\title{
GAS-PUFF TARGET FOR LASER-PRODUCING PLASMA SXR SOURCE
}

\author{
Š́́rka Salačováa,*, Przemyslaw Wachulak $^{b}$, Milan TatíčeK $^{a}$, \\ Miroslava Vrbovía \\ ${ }^{a}$ Czech Technical University in Prague, Faculty of Biomedical Engineering, Sitná 3105, Kladno, Czech Republic \\ ${ }^{b}$ Military University of Technology, Institute of Optoelectronics, gen. Sylwestra Kaliskiego 2 str., 00-908 \\ Warszawa 49, Poland \\ * corresponding author: sarka.salacova@fji.cvut.cz
}

\begin{abstract}
Mass density spatial distributions of selected gas-puff targets have been determined by radiography, using quasi-monochromatic radiation at a wavelength of $13 \mathrm{~nm}$. Results of spatial distributions of mass densities at various initial reservoir pressures for Nitrogen, Argon and Krypton targets are presented. Changes in the mass density spatial distribution due to a nozzle wear are reported.
\end{abstract}

KEYWORDS: Soft x-rays, gas-puff target, Nitrogen, Argon, Krypton.

\section{INTRODUCTION}

Our goal is to optimize the soft X-ray (SXR; photon energies of about $250 \mathrm{eV}$ to several $\mathrm{keV}$ [1]) source based on laser-plasma created by the focused laser radiation in the gas. If the intensity of the laser radiation is high enough, highly ionized ions are formed in the plasma and soft X-rays with the wavelength range $1-100 \mathrm{~nm}$ are emitted. The emitted radiation consists of a continuous spectral part due to inverse bremsstrahlung and line part of spectra due to spontaneous quantum transitions between inner states of ions. The higher the plasma electron temperature is, the shorter wavelengths are emitted.

The interest in laser-plasma sources is stimulated by potential innovative applications, where intensive sources of monochromatic radiation are required. If the objective is to develop SXR microscopy [2-4] of biological samples [5] 7], the preference is given to a radiation with a wavelength in the "water window" spectral range (i.e. $2.3-4.4 \mathrm{~nm}$ ). In this spectral range, a high contrast between the absorption of water and organic molecules containing carbon is achieved. In particular, when studying the Nitrogen plasma emission, it is possible to observe that a line emission at $2.88 \mathrm{~nm}$ dominates in plasma with an electron temperature about $\mathrm{eV}$ [8, 9]. To get a high intensity of this monochromatic radiation, a high density of Nitrogen ions is required. The high density of gas atoms in a defined region of space is achieved by the gas-puff target technique. The target forms a gas spreading into the vacuum after the nozzle is quickly opened. The spatial-temporal distribution of atomic density represents a spatial-temporal variable gas target. The efficiency of the SXR generation depends on the moment and place of the impact of the focused laser beam on the target.
In this paper, we present the results of an experimental determination of spatial densities of gas in our gas-puff target by radiography. The spatial dependence of the transmittance of a cylindrically symmetric shape perpendicular to symmetry axis has been measured using the quasi-monochromatic EUV radiation at a wavelength of $13.4 \mathrm{~nm}$ from another laser plasma-source. The experiments were performed in the cooperating laboratory of Laser-Matter Interaction Group, Institute of Optoelectronics, Military University of Technology in Warsaw [10].

\section{GAS-PUfF TARGET DESCRIPTION}

The gas-puff target presented herein was designed in Laser Laboratory Goettingen [11, 12] and is used in the CTU laboratory. A simple single-stream supersonic nozzle forms the gas target. The simplified scheme of the nozzle and target is seen in Fig. 1

The nozzle is a conical hole in a metal plate, located above a high-pressure reservoir and an evacuated space above the nozzle. At the beginning of the experiment, the gas pressure in the reservoir is increased almost immediately. The gas flows through the nozzle by supersonic speed. A variable gaseous target is formed above the nozzle, having, in principle, the cylindrical symmetry.

For our purpose, it is important to know especially the density of the target at the time when the laser is fired and at the place where the laser beam hits the target, i.e. at a distance $\Delta z$ from the nozzle.

\section{Description of gas stream RADIOGRAPHY}

Our gas-puff target has a cylindrical symmetry. The axis of symmetry coincides with our coordinate $z$. For the assessment of the spatial distribution of working gas mass density $\rho(x, y, z)$, we record the shadowgraph 


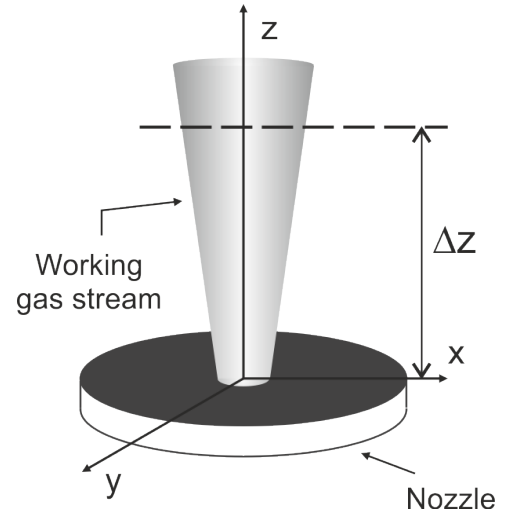

FIGURE 1. Scheme of gas-puff target.

of the gas stream in $y$-direction (perpendicular to the axis of symmetry $z$ ). The resulting $x-z$ dependence of the object radiography is determined by transmittance $T(x, z)$

$$
T(x, z)=e^{-\mu \int \rho(x, y, z) d y},
$$

where $\rho(x, y, z)$ is the mass density of the gas and $\mu$ is the photo-absorption cross-section of the gas at the wavelength used for the radiography experiment.

The recorded transmission along the $x$ coordinate at the distance $\Delta z$ is

$$
T(x, \Delta z)=e^{-\mu \int \rho(x, y, \Delta z) d y} .
$$

Assuming the Gaussian gas density profile $\rho(r, \Delta z)=\rho_{0}(\Delta z) \cdot e^{-\frac{r^{2}}{\sigma^{2}}}$, where $\sigma$ is the standard deviation of Gaussian function, we get transmittance:

$$
T_{G}(x, \Delta z)=e^{-\mu \int_{-\infty}^{\infty} \rho_{0}(\Delta z) \cdot e^{-\frac{\left(x^{2}+y^{2}\right)}{\sigma^{2}}} d y} .
$$

After integration, we found the transmittance function

$$
T_{G}(x, \Delta z)=e^{-\mu \rho_{0}(\Delta z) e^{-\frac{x^{2}}{\sigma^{2}} \sigma \sqrt{\pi}}} .
$$

The transmittance at the target axis (for $x=0$ ) allows us to state the gas mass density on the axis

$$
\rho_{0}\left(\Delta_{z}\right)=\frac{-1}{\mu \cdot \sigma \cdot \sqrt{\pi}} \ln T_{G}(0, \Delta z),
$$

if the standard deviation $\sigma$ is determined from the solution of the transcendent equation

$$
\frac{\ln T_{G}(\sigma, \Delta z)}{\ln T_{G}(0, \Delta z)}=e^{-1}
$$

\section{EXPERIMENTAL ARRANGEMENT}

Our scheme of the radiography experiment can be seen in Fig. 2. The investigated gas-puff target was located $640 \mathrm{~mm}$ away from the laser-plasma source and $155 \mathrm{~mm}$ from the CCD detector, all of them placed inside a vacuum chamber.

The source of radiation was laser-plasma created by Nd:YAG laser $(\lambda=1064 \mathrm{~nm}, E=450 \mathrm{~mJ})$ in $\mathrm{N} / \mathrm{He}$

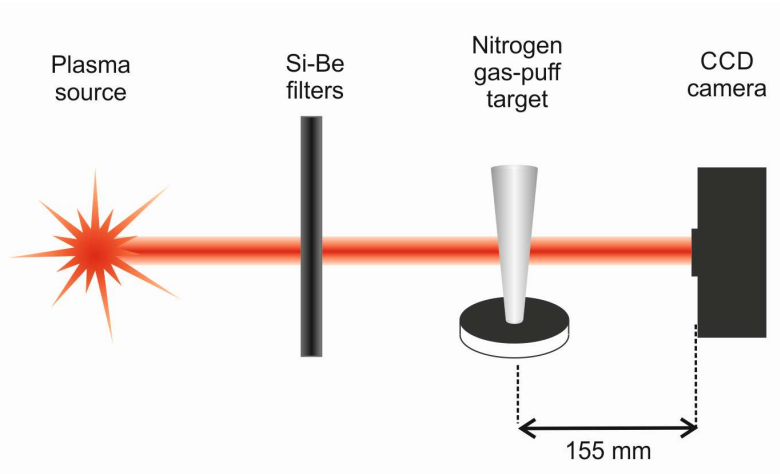

Figure 2. Principle scheme of the radiography experiment.

double gas-puff, then transmitted through a set of self-standing X-ray filters composed from $20 \mathrm{~mm}$ thick Si and $10 \mathrm{~mm}$ thick Be foils to get the wavelength of $13 \mathrm{~nm}$ 13. The CCD camera used was M25 X-Vision Rigaku, $512 \times 512$ square pixels $(24 \mu \mathrm{m})$, cooled by the Peltier element. The method was described in [13].

\section{RESUlts OF EXPERIMENTS}

\subsection{Results obtained with Nitrogen}

The spatial distribution of the energy of monochromatic radiation transmitted through our nitrogen gaspuff target in the proximity exposure is shown in Fig. 3. This radiography was taken at a time delay $\Delta t$, which corresponds to the time difference between opening the valve (the moment of filling the reservoir) and the time of exposure, which coincides with the time of plasma source emission, given by Nd:YAG laser shot. The gas-puff targets were backlit with EUV radiation. We get the shadowgraph that was discoloured for better viewing.

The logarithm of the object transmittance $T$ at the distance $\Delta z=0.5 \mathrm{~mm}$ from the nozzle aperture, evaluated from the digital record of the radiograph along the coordinate $x$ is shown in Fig. 3b. The measured values were taken as the average of the results of 5 rows and 5 shots. The recorded curve may be well fitted by the Gaussian curve and allows us to state the standard deviation of Gaussian distribution $\sigma=436 \mu \mathrm{m}$. If we also take into account the photo-absorption coefficient $\mu=5.3176 \cdot 10^{4} \mathrm{~cm}^{2} / \mathrm{g}$ for the wavelength $13.4 \mathrm{~nm}$ [14] we can evaluate the gas density on the axis as $\rho_{0}(\Delta z)=4.49 \cdot 10^{-4} \mathrm{~g} \cdot \mathrm{cm}^{-3}$.

\subsection{Results obtained With Argon and KRYPTON}

The method can be used to experimentally determine the mass density space distribution of any gas emerging from the nozzle. We have performed the measurements with Argon and Krypton passing through the same nozzle as shown in Fig. 4 A comparison of basic target parameters and their comparison with nitrogen-target ones can be seen in Table 1 . 


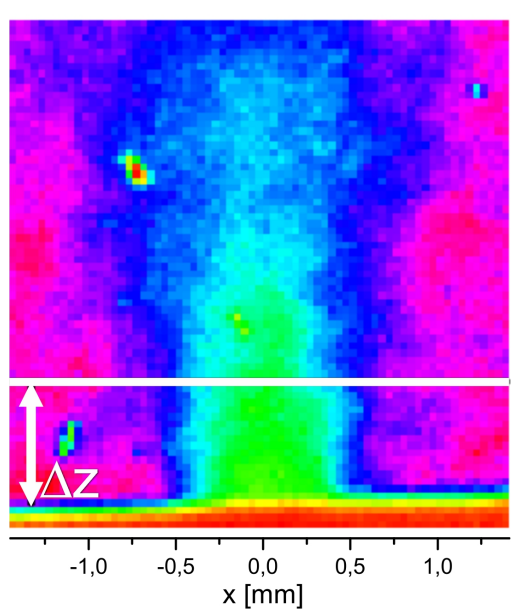

(A).

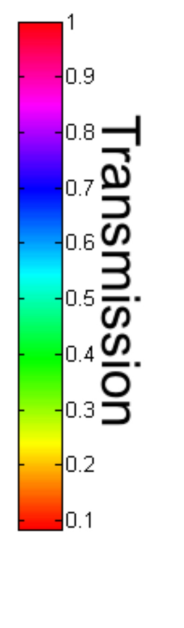

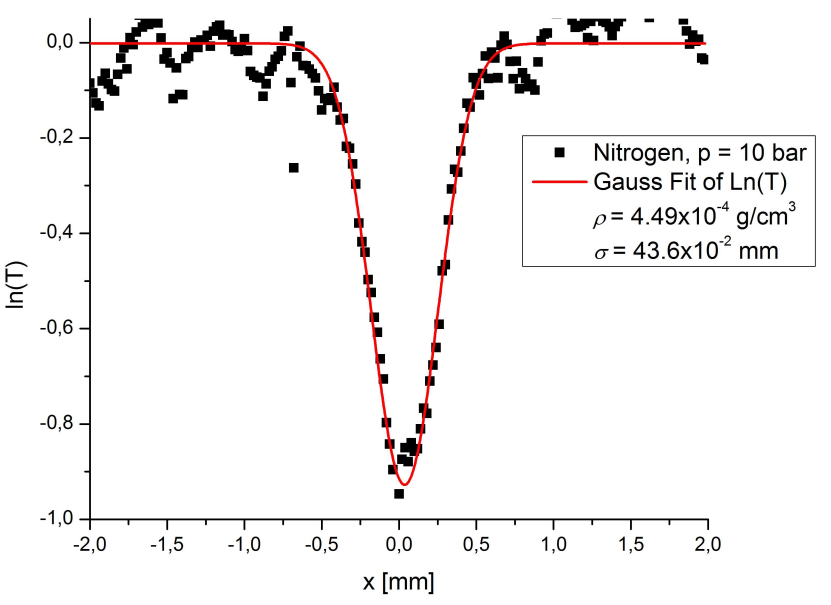

(B).

FiguRE 3. (a) Radiograph for nitrogen target; reservoir pressure $P_{0}=10$ bar, exposure time delay $\Delta t=900 \mu \mathrm{s}$. (b) Logarithm of nitrogen target transmittance at the distance $\Delta z=0.5 \mathrm{~mm}$ from the nozzle; the red line is the Gaussian fit.

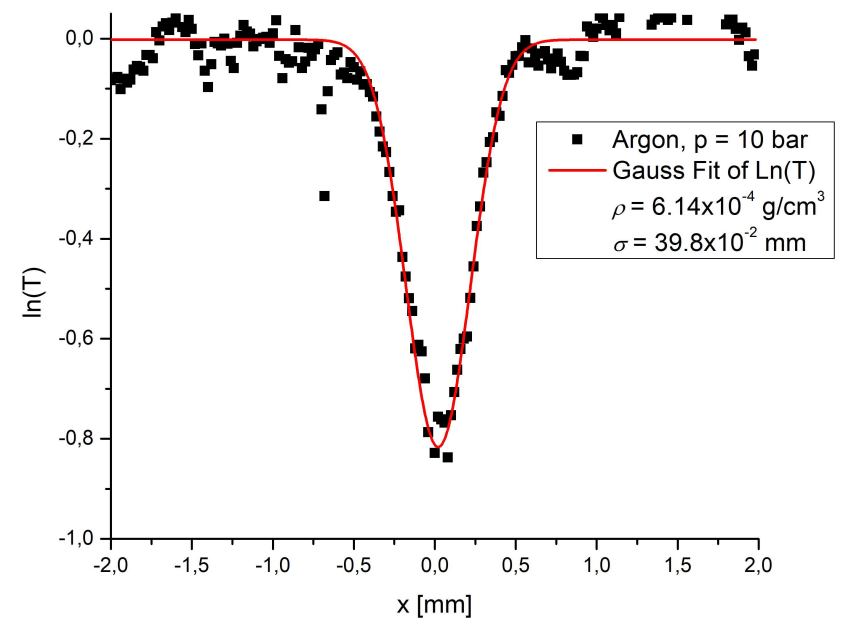

(A).

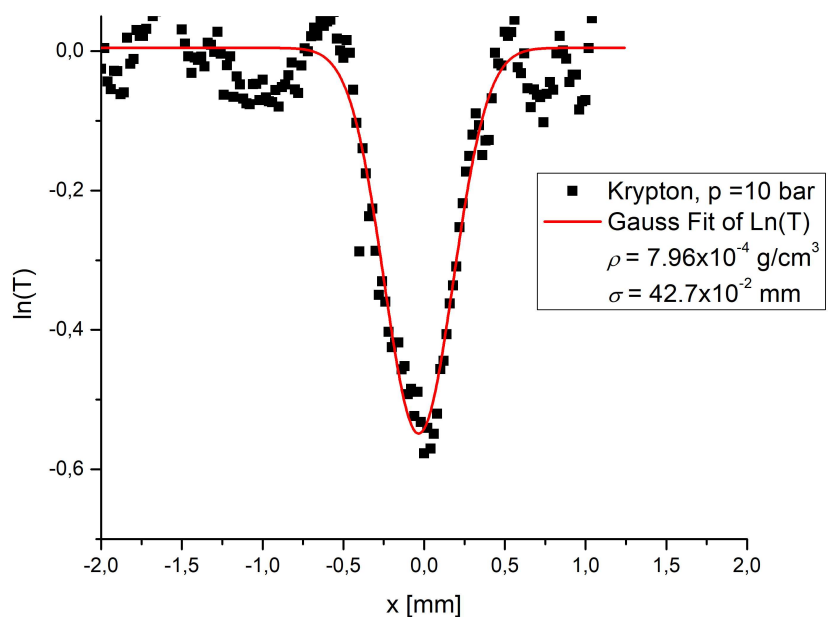

(B).

Figure 4. The profiles of logarithm of transmission for Argon (a) and Krypton (b) at distance $\Delta z=0.5 \mathrm{~mm}$, $P=10$ bar and exposure time delay $\Delta t=900 \mu \mathrm{s}$.

\begin{tabular}{lcccc}
\hline Gas & Atomic number & $\begin{array}{c}\text { Absorption coefficient } \\
\mu\left[\mathrm{cm}^{2} / \mathrm{g}\right]\end{array}$ & $\begin{array}{c}\text { Mass density } \\
\rho\left[\mathrm{g} / \mathrm{cm}^{3}\right]\end{array}$ & $\begin{array}{c}\text { Radial dimension } \\
\sigma[\mu \mathrm{m}]\end{array}$ \\
\hline Nitrogen & 7 & $5.3176 \times 10^{4}$ & $4.49 \times 10^{-4}$ & 436 \\
Argon & 18 & $2.0823 \times 10^{4}$ & $6.14 \times 10^{-4}$ & 398 \\
Krypton & 36 & $4.7344 \times 10^{4}$ & $7.96 \times 10^{-4}$ & 427 \\
\hline
\end{tabular}

TABLE 1. Gas-puff target parameters. 


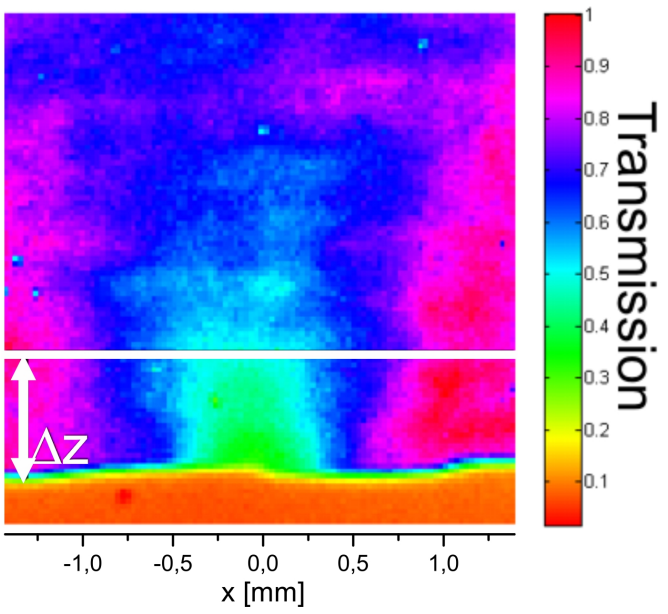

(A).

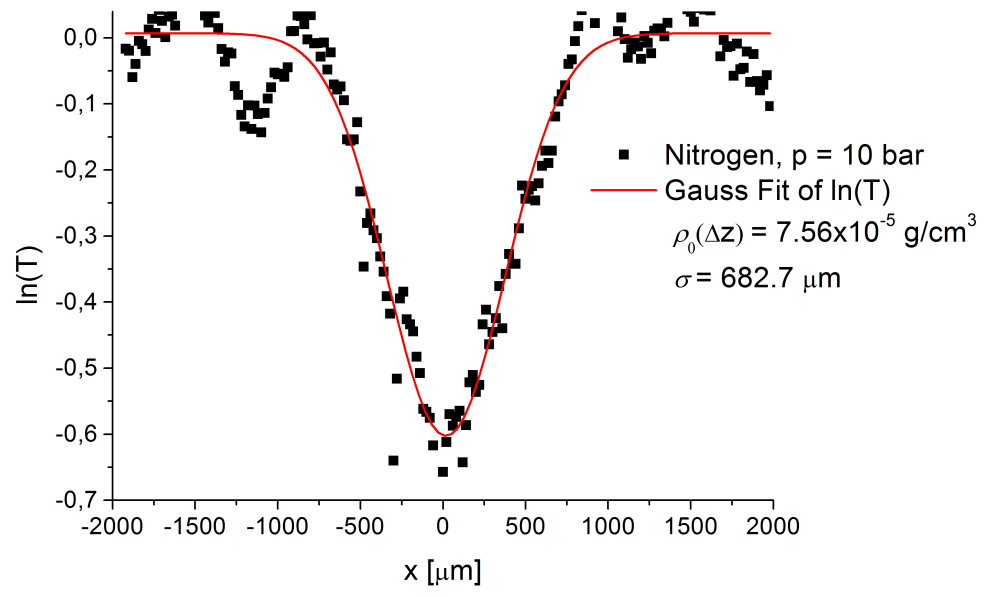

(в).

FiguRE 5. (a) Shadowgraph for our "worn-out" nozzle; reservoir nitrogen pressure $P_{0}=10 \mathrm{bar}, \Delta t=900 \mu \mathrm{s}$, (b) Logarithm of Nitrogen transmittance at the distance $\Delta z=0.5 \mathrm{~mm}$ The red line is the best Gaussian fit.

\subsection{NOZZLE AGEING}

If the nozzle is used for the SXR plasma generation, it is slightly ablated by the plasma created in its proximity during every laser shot. The profile of the nozzle is gradually changed and spatial mass density distribution of the gas target is also changed. We have aimed to compare the mass density for a new nozzle and "worn-out" nozzle.

The shadowgraph, shown as Fig. 5a was recorded with our "worn-out" nozzle, which was previously used in the SXR source system for several thousand shots. The Fig. 5b shows the corresponding spatial dependence of transmittance of the gas-puff target at the distance $\Delta z=0.5 \mathrm{~mm}$ from this nozzle. The estimated gas density on the axis $\rho_{0}(\Delta z)=0.76 \times 10^{-4} \mathrm{~g} \cdot \mathrm{cm}^{-3}$ is 5 times lower and the gas jet is significantly wider than that observed with our new nozzle.

\subsection{Pressure Dependence of MASS DENSITY}

The spatial distribution of the gas target mass density depends also on the gas reservoir pressure. We have repeated the experiments with nitrogen for various pressures $P_{0}$ and for both new and "worn-out" nozzles.

The results can be seen in Fig. 6. The "worn-out" nozzle provides a reasonably lower peak value of the Nitrogen density at the same place of the stream.

For a given nozzle profile, the gas density increases with increasing initial (reservoir) pressure, but saturation effect is verified above a certain pressure level. The value of the pressure saturation threshold is affected by the nozzle geometry.

\section{Conclusions}

The gas-puff target for the laser-producing plasma SXR source was investigated by the EUV radiography. We were studying the spatial distribution of the mass density in the gas-puff target for three different gasses and also the development of the gas-puff target in time. As working gasses, we used Argon, Krypton and Nitrogen for their property to radiate in the water window.

The mass density for each gas at pressure $P=$ 10 bar (Table 1) shows that the density of Krypton is almost twice that of Nitrogen. It is also possible to observe that the geometry of the gas-puff target is very similar for all gasses and it mostly depends on the nozzle geometry. In our case, it was a conical nozzle with an output diameter of $0.54 \mathrm{~mm}$. The radial dimension $\sigma$ in the investigated gas-puff target at a distance of $\Delta z=0.5 \mathrm{~mm}$ was around $0.4 \mathrm{~mm}$. We will use all these results as the input data for the plasma simulation to find the optimal setup for our SXR source. We can also compare them with other nozzle types to find the optimal shape (e.g., with the double stream nozzle which is used in the laboratory of Laser-Matter Interaction Group, Institute of Optoelectronics, Military University of Technology in Warsaw [15]).

In chapter 5.3 we have shown the problem of nozzle ageing. Thanks to the formation of plasma, the nozzle is being damaged and the gas-puff target is changing. The mass density decreases at a defined distance $\Delta z$ that also leads to changes in the formation of plasma. The differences between mass density for new and old nozzles are shown in Figure6. Thanks to these results, we have understood how the spatial target parameters are changed during a long-term use.

We also compared how the mass density depends on the input pressure. In Figure 6, we can see that if the pressure equals or is higher than $P=10$ bar, the mass density is stable for the new nozzle and an increase in gas pressure will not result in an increase in mass density.

If the target density is known, we may proceed with the computer optimization of our system via laser parameter variation. Moreover, further nozzle opti- 


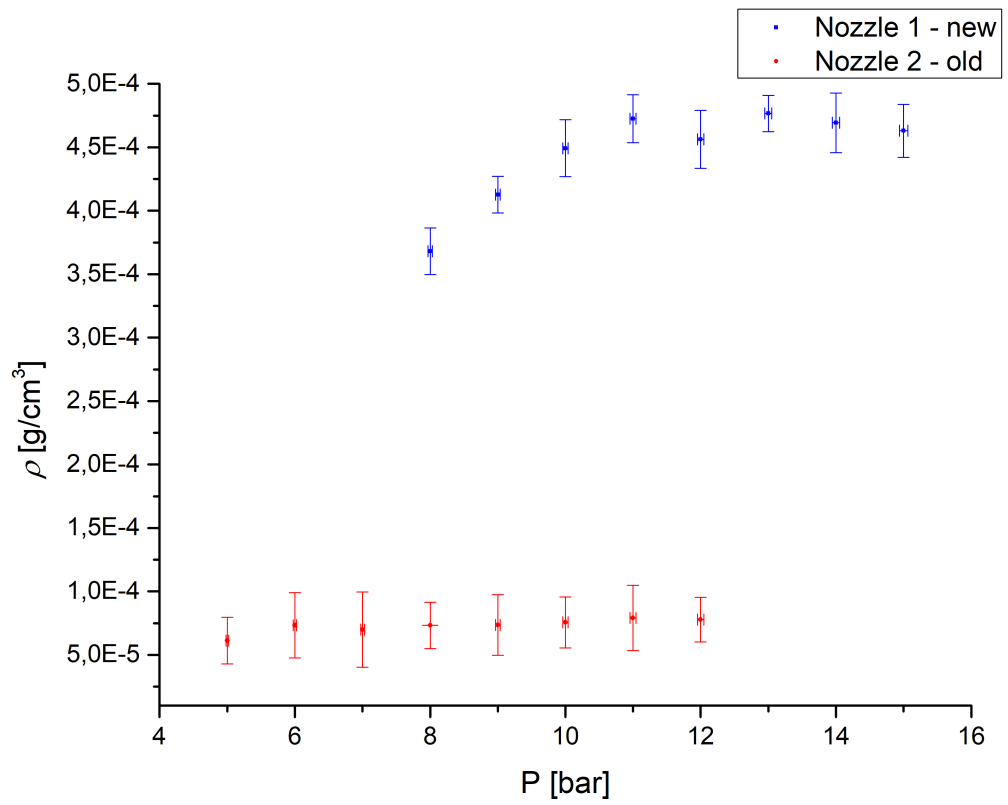

FIGURE 6. Nitrogen mass densities at the nozzle axis versus reservoir pressure at $\Delta t=900 \mu \mathrm{s}$ and $\Delta z=0.5 \mathrm{~mm}$ for new nozzle 1 and "worn-out" nozzle 2. $P$ is Nitrogen reservoir pressure, $\rho_{0}$ is mass density in the gas stream. Blue spots correspond with values for new (first time used) nozzle, the red spots correspond with values for the old nozzle (after 1000 use).

mization may also be performed via target computer modelling when these experimental results are used to validate the computer model.

\section{ACKNOWLEDGEMENTS}

This work was supported by grants on the projects of MEYS CR in the frame of the program INTER Excellence on the project LTT17015 and SGS13/094/OHK4/1T/17 Optimization of XUV radiation sources for biological imaging.

\section{REFERENCES}

[1] D. Attwood. Soft X-Rays and Extreme Ultraviolet Radiation: Principles and Applications. Cambridge University Press, Cambridge, 1999. DOI:10.1017/CBO9781139164429

[2] A. Bayer, F. Barkusky, S. Döring, et al. Applications of compact laser-driven EUV/XUV plasma sources. $X$-Ray Optics and Instrumentation 2010, 2009. DOI:10.1155/2010/687496

[3] P. W. Wachulak, A. Bartnik, M. Skorupka, et al. Water-window microscopy using a compact, laser-plasma SXR source based on a double-stream gas-puff target. Applied Physics B 111(2):239-247, 2013. DOI:10.1007/s00340-012-5324-y

[4] H. M. Hertz, M. Berglund, G. A. Johansson, et al. Compact water-window x-ray microscopy with a droplet laser-plasma source. AIP Conference Proceedings 507(1):721-725, 2000. DOI:10.1063/1.1291239

[5] A. Torrisi, P. Wachulak, M. Nawaz, et al. Applications of a compact "water window" source for investigations of nanostructures using sxr microscope. Acta Physica Polonica A 129:169-171, 2016. DOI:10.12693/APhysPolA.129.169.
[6] J. Kirz, C. Jacobsen, M. Howells. Soft x-ray microscopes and their biological applications. Quarterly Reviews of Biophysics 28(1):33-130, 1995. DOI:10.1017/S0033583500003139

[7] P. Wachulak, A. Bartnik, H. Fiedorowicz. Nanoimaging using a compact laser plasma soft $\mathrm{x}$-ray source based on a gas puff target. In B. Lai, A. Somogyi (eds.), X-Ray Nanoimaging: Instruments and Methods IV, vol. 11112, pp. 117 - 123. International Society for Optics and Photonics, SPIE, 2019. DOI:10.1117/12.2529468.

[8] P. Vrba, M. Vrbová, P. Brůža, et al. XUV radiation from gaseous nitrogen and argon target laser plasmas. Journal of Physics: Conference Series 370:357 - 364, 2012. DOI:10.1088/1742-6596/370/1/012049

[9] P. Vrba, M. Vrbova, S. V. Zakharov, V. S. Zakharov. Modeling of ns and ps laser-induced soft X-ray sources using nitrogen gas puff target. Physics of Plasmas 21(7):073301, 2014. DOI:10.1063/1.4887295

[10] Instytut optoelektroniki WAT. Www.ioe.wat.edu.pl/ 2013. Accessed: 27 October 2019.

[11] S. Kranzusch, K. Mann. Spectral characterization of EUV radiation emitted from a laser-irradiated gas puff target. Optics Communications 200(1):223 - 230, 2001. DOI:10.1016/S0030-4018(01)01639-X

[12] S. Vondrova, M. Vrbova, D. Pánek, et al. Diagnostics and modeling of gas puff target laser plasma radiation source. In 2012 International Workshop on EUV and Soft X-Ray Sources, p. 11. EUV Litho Inc, Austin, 2012.

[13] R. Rakowski, A. Bartnik, H. Fiedorowicz, et al. Pulsed X-ray radiography of a gas jet target for laser-matter interaction experiments with the use of a CCD detector. Nuclear Instruments and Methods in Physics Research Section A, Accelerators, Spectrometers, Detectors and Associated Equipment 551:139 - 144, 2005. DOI:10.1016/j.nima.2005.07.047 
[14] X-ray properties of the elements. http://henke.lbl gov/optical_constants/pert_form.html 1997.

Accessed: 14 June 2019.

[15] P. W. Wachulak, A. Torrisi, A. Bartnik, et al.

Desktop water window microscope using a

double-stream gas puff target source. Applied Physics B

118(4):573-578, 2015. DOI:10.1007/s00340-015-6044-x. 